PROCEEDINGS OF THE

AMERICAN MATHEMATICAL SOCIETY

Volume 33, Number 2, June 1972

\title{
ON THE RADICAL OF THE GROUP ALGEBRA OF A $p$-GROUP OVER
}

\section{A MODULAR FIELD}

\author{
GAIL L. CARNS AND CHONG-YUN $\mathrm{CHAO}^{1}$
}

\begin{abstract}
Let $G$ be a finite $p$-group, $K$ be the field of integers modulo $p, K G$ be the group algebra of $G$ over $K$ and $N$ be the radical of $K G$. By using the fact that the annihilator, $A(N)$, of $N$ is one dimensional, we characterize the elements of $A\left(N^{2}\right)$. We also present relationships among the cardinality of $A\left(N^{2}\right)$, the number of maximal subgroups in $G$ and the number of conjugate classes in $G$. Theorems concerning the Frattini subalgebra of $N$ and the existence of an outer automorphism of $N$ are also proved.
\end{abstract}

1. Introduction. Throughout this note, we let $p$ be a prime, $G$ be a finite $p$-group, $K$ be the field of integers modulo $p$ and $K G$ be the group algebra of $G$ over $K$. It is well known that $K G$ is not semisimple; the fundamental ideal $N=\left\{\sum_{g \in G} \alpha_{g} g \in K G ; \sum_{g \in G} \alpha_{g}=0\right\}$ of $K G$ is its radical ([3], [6]). Let $e$ be the identity of $G$, then the elements $g-e$ for all $g \neq e$ in $G$ constitute a basis for $N$. Hence, the dimension, $\operatorname{dim} N$, of $N$ is equal to $|G|-1$ where $|G|$ is the order of $G$. Also, $K G$ is the semidirect sum of the ideal $N$ and the subalgebra $\langle e\rangle$. The nilpotent associative algebra $N$ is said to be of exponent $t$ if $N^{t} \neq 0$ and $N^{t+1}=0$, i.e.,

$$
N=N^{1} \supset N^{2} \supset \cdots=N^{t} \supset N^{t+1}=0 .
$$

Recently, Hill in [2] showed that the annihilator (two sided) of $N^{i}, A\left(N^{i}\right)$, is $N^{t+1-i}, 1 \leqq i \leqq t$. In this note we shall present some properties of $N$ by centering around the fact that $A(N)$ is isomorphic to $K$, i.e., the dimension of $A(N)$ is one. In $\S 2$, we present a characterization of elements in $A\left(N^{2}\right)$ and relationships among the cardinality, $\left|A\left(N^{2}\right)\right|$, of $A\left(N^{2}\right)$, the number of maximal subgroups of $G$ and the number of conjugate classes in $G$. In particular, $\operatorname{dim} A\left(N^{2}\right)$ is equal to the least number of generators of $G$ plus one. In $\S 3$, we show that the Frattini subalgebra of any associative nilpotent algebra $U$ over a field is $U^{2}$. We also use Stitzinger's results in [7]

Received by the editors September 2, 1971.

AMS 1970 subject classifications. Primary 16A26; Secondary 16A22.

Key words and phrases. Modular group algebra, radical, annihilator, center, dimension, maximal subgroups, conjugate classes, Frattini subalgebra, nonimbedding, outer automorphism.

${ }^{1}$ The authors wish to thank the referee for his helpful suggestions.

(c) A merican Mathematical Society 1972 
to state the nonimbedding properties of $N$. In $\S 4$, analogous to Gaschütz' result in [1] on the existence of an outer $p$-automorphism of a finite nonabelian $p$-group, we show that $N$ has an automorphism of order $p$ which is not inner if $|G|>2$.

2. A characterization of elements in $A\left(N^{2}\right)$. For each element $\alpha=\sum_{g \in G} \alpha_{g} g \in K G$, we may associate a map $\alpha$ from $G$ to $K$ defined by $\alpha(g)=\alpha_{g}$. Clearly, this correspondence between $\alpha$ and $\alpha$ is one-to-one. Also, the addition of two such maps is defined as pointwise, i.e., $(\alpha+\beta)(g)=\alpha(g)+\beta(g)$. Let $N$ be the fundamental ideal of exponent $t$ in $K G$. Then, by Hill's result in [2], we know $A(N)=N^{t}$. Also, one can easily verify that $k \in A(N)=N^{t}$ if and only if $\boldsymbol{k}$ is a constant map, i.e., $k(g)=k$ for every $g \in G$ and $N^{t}=\left\langle\left(\sum_{g \in G} g\right)\right\rangle$.

THeOREM 1. Let $N$ be the fundamental ideal of exponent $t>1$ in KG and $\operatorname{Hom}\left(G, K^{+}\right)$be the set of group homomorphisms of $G$ into the additive group $K^{+}$of the integers modulo $p$. Then $\alpha \in A\left(N^{2}\right)$ if and only if $\alpha=\alpha^{*}+k$ for some $\alpha^{*} \in \operatorname{Hom}\left(G, K^{+}\right)$and some constant map $\boldsymbol{k}$. Further, $\alpha^{*}$ and $\boldsymbol{k}$ are unique for $\alpha$.

Proof. If $\alpha=\alpha^{*}+k$ for some $\alpha^{*} \in \operatorname{Hom}\left(G, K^{+-}\right)$and some constant map $k$, then for every $g \in G$, we have

$$
\alpha^{*}(g)=\alpha(g)-k(g)=x_{g}-k .
$$

Also, by using (1) and $\alpha^{*}(g h)=\alpha^{*}(g)+\alpha^{*}(h)$, we have

$$
\alpha_{g h}=\alpha_{g}+\alpha_{h}-k
$$

for all $g, h \in G$. Now by using (2), for all $h, u \in G$, we have

$$
\begin{aligned}
(h-e)(u-e) \alpha & =(h u-h-u+e)\left(\sum_{g \in G} \alpha_{g} g\right) \\
& =\sum_{g \in G}\left(x_{g} h u g-\alpha_{g} h g-\alpha_{g} u g+\alpha_{g} g\right) \\
& =\sum_{g \in G}\left(x_{u^{-1} h^{-1} g}-\alpha_{h^{-1} g}-\alpha_{u^{-1} g}+\alpha_{g}\right) g \\
& =\sum_{g \in G}\left[\left(\alpha_{u^{-1}}+\alpha_{h^{-1} g}-k\right)-\alpha_{h^{-1} g}-\left(\alpha_{u^{-1}}+\alpha_{g}-k\right)+\alpha_{g}\right] g \\
& =0
\end{aligned}
$$

Similarly, $\alpha(h-e)(u-e)=0$. It follows that $\alpha \in A\left(N^{2}\right)$.

Conversely, if $x \in A\left(N^{*}\right)$, then for all $h, u \in G$,

$$
0=\left(h^{-1}-e\right)\left(u^{-1}-e\right)\left(\sum_{g \in G} \alpha_{g} g\right)=\sum_{g \in G}\left(\alpha_{u h g}-\alpha_{h g}-\alpha_{u g}+\alpha_{e}\right) g .
$$


In particular, the coefficient of $e$ is zero, i.e.,

or

$$
\alpha_{u k}=\alpha_{u}+\alpha_{h}-\alpha_{e},
$$

$$
\alpha(u h)=\alpha(u)+\alpha(h)-\alpha_{e} .
$$

Let $k=\alpha_{e}$ and $\alpha^{*}=\alpha-k$, then (3) can be written as

$$
\alpha^{*}(u h)=\alpha^{*}(u)+\alpha^{*}(h),
$$

i.e., $\alpha^{*} \in \operatorname{Hom}\left(G, K^{+}\right)$.

The uniqueness follows from the fact that $\alpha^{*}(e)=0$ yields $\alpha(e)=k(e)$.

REMARK. By Hill's result in [2], in Theorem 2, $A\left(N^{2}\right)$ can be replaced by $N^{t-1}$.

COROLlaRY 1.1. Let $r=\operatorname{dim} A\left(N^{2}\right)=\operatorname{dim} N^{t-1}, \quad m=$ the number of maximal subgroups of $G, d=$ the least number of elements which generate $G$, $c=$ the number of conjugate classes in $G$ and $\phi(G)=$ the Frattini subgroup of $G$. Then,

(i) $\left|A\left(N^{2}\right)\right|=p \cdot|(G / \phi(G))|$,

(ii) $m=\sum_{i=0}^{r-2} p^{i}$,

(iii) $r=d+1$,

(iv) $G$ is cyclic if and only if $r=2$,

(v) $G$ is elementary abelian if and only if $r=n+1$ where $|G|=p^{n}$,

(vi) $m=\sum_{i=0}^{d-1} p^{i}$,

(vii) $A\left(N^{2}\right)=N^{t-1} \subseteq Z(N)$ where $Z(N)$ is the center of $N$,

(viii) $m \leqq \sum_{i=0}^{c-4} p^{i}$ if $|G|>4$.

Proof. (i) By Theorem $1,\left|A\left(N^{2}\right)\right|=p \cdot\left|\operatorname{Hom}\left(G, K^{+}\right)\right|$. Since $K^{+}$is a simple group, the kernel of any nonzero map $\eta$ in $\operatorname{Hom}\left(G, K^{+}\right)$is a maximal subgroup in $G$. Since the kernel of $\eta$ contains the kernel of the natural map from $G$ onto $G / \phi(G)$, any homomorphism of $G$ into $K^{+}$can be factored through $G / \phi(G)$. Thus, $\left|\operatorname{Hom}\left(G, K^{+}\right)\right|=\left|\operatorname{Hom}\left(G / \phi(G), K^{+}\right)\right|$. Also, $G / \phi(G)$ is elementary abelian and every finite abelian group is isomorphic to its dual group [5, p. 50], therefore we have

$$
\left|\operatorname{Hom}\left(G / \phi(G), K^{+}\right)\right|=|G / \phi(G)| \text {. }
$$

Consequently,

$$
\left|A\left(N^{2}\right)\right|=p \cdot\left|\operatorname{Hom}\left(G, K^{+}\right)\right|=p \cdot|G / \phi(G)| .
$$

(ii) Let $\sigma$ be a nonzero homomorphism of $G$ onto $K^{+}$. Then the kernel of $\sigma$ is a maximal subgroup of $G$. Two nonzero homomorphisms in $\operatorname{Hom}\left(G, K^{+}\right)$have the same kernel if and only if they differ by an automorphism of $K^{+}$. Thus, $\left|\operatorname{Hom}\left(G, K^{+}\right)\right|=1+(p-1) m$ and $p^{r}=\left|A\left(N^{2}\right)\right|=$ $p$ Hom $\left|\left(G, K^{+}\right)\right|=p(1+(p-1) m)$, i.e., $m=\sum_{i=0}^{r-2} p^{i}$. 
(iii) By (i), $r=\operatorname{dim}\left(A\left(N^{2}\right)\right)=\operatorname{dim}_{K}(G / \phi(G))+1$ and, by the Burnside basis theorem, $\operatorname{dim}_{K}(G / \phi(G))=d$.

(iv), (v) and (vi) follow from (i), (ii) and (iii).

REMARK. By using Corollary 14 in [2] we can state: If $r=2, K G$ has exactly one ideal of each dimension.

(vii) It is well known that the conjugate sums $C^{1}=e, C^{2}, \cdots, C^{c}$ constitute a basis for the center, $Z(K G)$, of $K G$ where each $C^{i}$ is the sum of elements in a conjugate class in $G$. Let $\alpha=\sum_{o \in G} \alpha_{g} g$ be an arbitrary element in $A\left(N^{2}\right)$. If $u$ and $h$ are conjugates in $G$, i.e., $h=v u v^{-1}$ for some $v \in G$, then, by using Theorem 1 , we have

$$
\alpha_{h}=\alpha^{*}(h)+k=\alpha^{*}\left(v u v^{-1}\right)+k=\alpha^{*}(u)+k=\alpha_{u} .
$$

Hence, $\alpha$ is a linear combination of conjugate sums, i.e., $\alpha \in Z(K G)$. Since $Z(N)=Z(K G) \cap N, A\left(N^{2}\right) \subseteq Z(N)$.

(viii) Since $Z(N)=Z(K G) \cap N$ and $e \in Z(K G)$ and $e \notin N, \operatorname{dim} Z(N)<$ $\operatorname{dim} Z(K G)=c$. Let $a_{i}, 2 \leqq i \leqq c$, be the cardinality of the conjugate class from which the sum $c^{i}$ is taken. We note that since $G$ is a $p$-group, $a_{i}$ is equal to a power of $p$ greater than one if the conjugate class consists of more than one element. Since $C^{1}, C^{2}, \cdots, C^{c}$ constitute a basis for $K G$, $C^{2}-a_{2} e, C^{3}-a_{3} e, \cdots, C^{c}-a_{c} e$ are in $Z(N)$ and are linearly independent. Hence, $\operatorname{dim} Z(N)=c-1$.

Since $G$ is a $p$-group, there is a nonidentity $h$ in $Z(G)$ such that $h-e \notin N^{t-1}$. The reason is that if $h-e$ belonged to $N^{t-1}$, then $(u-e)(h-e)=$ $\sum_{g \in G} g$ for some $u \in G$. This is impossible since $|G|>4$. Consequently, $A\left(N^{2}\right) \neq Z(N)$ and $p(1+(p-1) m)=\left|A\left(N^{2}\right)\right|<p^{c-1}$, i.e., $p(1+m(p-1)) \leqq$ $p^{c-2}$, and $m \leqq\left(p^{c-3}-1\right) /(p-1)=\sum_{i=0}^{c-4} p^{i}$.

REMARK. If $G$ is the dihedral group of order 8 and if $K$ is the field of integers modulo 2, then $m=3, c=5$ and the equality in (viii) holds.

3. Nonimbedding. Let $S$ be an associative algebra (not necessarily finite dimensional) over a field. The Frattini subalgebra, $\phi(S)$, of $S$ is defined as the intersection of all maximal subalgebras of $S^{\prime}$ if maximal subalgebras of $S^{\prime}$ exist and as $S$ otherwise. Stitzinger sinowed in [7, p. 531] that if $B$ is a nontrivial finite dimensional nilpotent associative algebra over a field such that the right annihilator of $B$ is one dimensional, then $B$ cannot be imbedded as an ideal in any associative algebra $S$ contained in $\phi(S)$.

THEOREM 2. Let $U$ be a nilpotent associative algebra over a field $F$. Then $\phi(U)=U^{2}$.

In order to prove Theorem 2, we need the following: We define the normalizer, $n_{V}(W)$, of a subalgebra $W$ in an associative algebra $V$ over a field $F$ to be $\{v \in V: v W \subseteq W$ and $W v \subseteq W\}$. We say that a subalgebra $W$ is self-normalizing if $n_{V}(W)=W$. 
LEMMA 1. Let $V$ be a nilpotent associative algebra of exponent $t>1$ over a field $F$. If $W$ is a proper subalgebra of $V$ then $W$ is not self-normalizing.

Proof. $W$ contains $V^{t+1}=0$. Assume that $W$ contains $V^{j}$ and does not contain $V^{j-1}$. Then $W+V^{j} \subseteq W$ and $W+V^{j-1} \varsubsetneqq W$. Aiso,

$\left(W+V^{j-1}\right) W \subseteq W+V^{j} \subseteq W$ and $W\left(W+V^{j-1}\right) \subseteq W+V^{j} \subseteq W$.

Hence, $n_{V}(W) \geqq W$.

The proof of Theorem 2 goes as follows: We claim that $U^{2} \supseteq \phi(U)$. Since $U / U^{2}$ has zero multiplication, every maximal subspace $\bar{M}_{\alpha}$ of the vector space $U / U^{2}$ is a maximal subalgebra. Hence $M_{\alpha}+U^{2}$ is a maximal subalgebra in $U$ and $U^{2} \supseteq \phi(U)$.

Now we show that $\phi(U) \supseteq U^{2}$. Let $M$ be any maximal subalgebra of $U$. By Lemma $1, M$ is an ideal in $U$. Hence, $\bar{U}=U / M \neq \overline{0}$. Since $M$ is maximal and $U$ is nilpotent, $\bar{U}$ is a nilpotent algebra with no proper subalgebras. Since $\bar{U}^{2}$ is a subalgebra of $\bar{U}$ and $\bar{U}$ is nilpotent, $\bar{U}^{2}=0$, i.e., $U^{2} \subseteq M$ for any arbitrary maximal subalgebra $M$ of $U$. It follows that $U^{2} \subseteq \phi(U)$.

COROLlaRY 2.1. Let $N$ be the fundamental ideal of $K G$ where $|G|>2$. Then $N$ cannot be imbedded as an ideal in any finite nilpotent associative algebra $B$ over $K$ such that $B^{2} \supseteq N$.

Proof. It follows from $\operatorname{dim} A(N)=1$, Stitzinger's result in [7] and our Theorem 2.

4. Outer automorphisms. Let $R$ be a ring with an identity $e$, then, for a right quasi-regular element $a$ in $R, \omega_{a}(x)=x+a^{\prime} x+x a+a^{\prime} x a=$ $\left(e+a^{\prime}\right) x(e+a)$, where $a^{\prime}$ is a right quasi-inverse of $a$, is an automorphism of $R$ called an inner automorphism of $R$. As indicated on p. 55 in [4], the algebra which has a basis $\{x, y, z\}$ over the field of integers modulo 2 with the multiplication defined by $x y=z$ and all other products being zero has no outer (noninner) automorphism. Since every nilpotent element is right quasi-regular and since $N$ is a nilpotent ideal in $K G$, for each $q \in N$, $\omega_{q}(x)=\left(e+q^{\prime}\right) x(e+q)$ is an inner automorphism of $N$. In fact, each automorphism $\bar{\omega}$ of $G$ induces an automorphism $\omega$ on $N$ defined linearly by $\omega\left(\sum_{g \in G} \alpha_{g} g\right)=\sum_{g \in G} \alpha_{\rho}(\bar{\omega} g)$. If $\bar{\sigma}_{g}(h)=g^{-1} h g$ is an inner automorphism of $G$, then one can easily verify that it induces an automorphism on $N$ which is equal to the inner automorphism $\omega_{g-e}$ on $N$. Although Gaschütz showed in [1] that every nonabelian $p$-group $G$ possesses a noninner automorphism whose order is a power of $p$, it is not known whether this outer automorphism of $G$ induces an outer automorphism on $N$. However, by using $A(N)=\left\langle\left(\sum_{g \in G} g\right)\right\rangle$ we can prove the following

THEOREM 3. Let $N$ be the fundamental ideal of $K G$ where $|G|>2$. Then $N$ has an automorphism of order $p$ which is not inner. 
Proof. Let $h \in G,(h-e) \in N$ and $(h-e) \notin N^{2}$. Since $(h-e) \notin N^{2}$, we may choose a complementary subspace $M$ of $\langle(h-e)\rangle$ in $N$ such that $M \supseteq N^{2}$. Then $N=M+\langle(h-e)\rangle$ where the sum is the direct sum of vector spaces. Since $|G|>2, z=\sum_{g \in G} g \in N^{2} \subseteq M$ and $M \neq 0$. Since every element $x \in N$ can be uniquely written as $x=y+k(h-e)$ where $y \in M$ and $k \in K$, we can define a linear transformation $T$ on $N$ such that $T y=y$ and $T(k(h-e))=k(\bar{h}-e)+$ $k z$. We claim that $T$ is an automorphism. By using $z \in A(N)$ and $M$ being an ideal in $N$ (since $M \supseteq N^{2}$ ), it follows that $T$ is an endomorphism. Also, $T(y+k(h-e)-k z)=y+k(h-e)$ indicates that $T$ is surjective. Hence, $T$ is an automorphism.

We claim that $T$ is not inner. Suppose the contrary, i.e., there existed a $q \in N$ such that $T=\omega_{q}$, then, we would, in particular, have

(4) $(h-e)+z=T(h-e)=\omega_{q}(h-e)=\left(e+q^{\prime}\right)(h-e)(e+q)$.

Multiplying both sides of (4) by $(e+q)$, we obtain

$$
(h-e)+z+q(h-e)=(h-e)+(h-e) q,
$$

i.e., $z=h q-q h$. Say $q=\cdots+x_{h^{-1}} h^{-1}+\cdots$, then $z=\left(\alpha_{h^{-1}}-\alpha_{h^{-1}}\right) e+\cdots$. But $z=\sum_{g \in G} g$. Hence, it is a contradiction, and $T$ is not inner.

Since $T^{p}(x)=T^{p}(y+k(h-e))=y+k(h-e)+p k z=x$ for every $x \in N, T$ is of order $p$.

\section{REFERENCES}

1. W. Gaschütz, Nichtabelsche p-Gruppen besitzen äussere p-Automorphismen, J. Algebra. 4 (1966), 1-2. MR 33 \#1365.

2. E. T. Hill, The annihilator of radical powers in the modular group ring of a p-group, Proc. Amer. Math. Soc. 25 (1970), 811-815. MR 41 \#6995.

3. S. A. Jennings, The structure of the group ring of a p-group over a modular field, Trans. Amer. Math. Soc. 50 (1941), 175-185. MR 3, 34.

4. R. L. Kruse and D. T. Price, Nilpotent rings, Gordon and Breach, New York, 1969. MR 42 \#1858.

5. Serge Lang, Algebra, Addison-Wesley, Reading, Mass., 1965. MR 33 \#5416.

6. Gerald Losey, On group algebras of p-groups, Michigan Math. J. 7 (1960), 237-240. MR 23 \#A212.

7. E. L. Stitzinger, A nonimbedding theorem of associative algebras, Pacific J. Math. 30 (1969), 529-531. MR 40 \#7307.

Department of Mathematics, University of Pittsburgh, Pirtsburgh, PenNSYLVANIA 15213. 